\title{
Bewusstsein als Verhängnis: Philosophieinterne Krisen
}

Seine Arbeit sei »aus den geistigen und politischen Kämpfen dieser Zeit entstanden « (Seidel 1927: 72). Alfred Seidel hat mit seinem Text Bewußtsein als Verhängnis einen Text verfasst, der Krisen - und in der hier eingenommenen Interpretation insbesondere philosophieinterne Krisen - am Anfang des 2o. Jahrhunderts in markanter Form nachzeichnet. Seidel, so Hugo Velarde, trage nicht nur zur Zeitdiagnose bei, sondern »ist selbst der untergehende Beweis für eine Zeit, die Verhängisvolles bereithält« (Velarde 2008: 176). Im Kontrast zu den drei folgenden Fallbeispielen (Husserl, Wiener Kreis, Kritische Theorie) verkörpern Seidel und sein Text im vorliegenden Buch jene Krisen, die Philosophen erkenntnistheoretisch zu bewältigen versuchen.

Nebst Velarde hat sich in jüngerer Zeit der Kulturhistoriker Christian Voller in Form zweier Aufsätze intensiv mit Seidel beschäftigt. Für die hier geführten Auseinandersetzungen sind Vollers wissenschaftshistorische Aufarbeitungen instruktiv, d.h., die biografischen und wissenschaftshistorischen Angaben über Seidel sowie das Bild, das rezeptionsgeschichtlich von ihm gezeichnet wurde, sind vor allem seinen Aufsätzen entnommen. Eine Besonderheit an Seidels Text ist dessen Edition: Wie erwähnt ist es der Psychiater und Kunstwissenschaftler Hans Prinzhorn, der Seidels Buch nach dessen Suizid posthum herausgibt. Es bleibt unklar, »welchen Anteil Prinzhorn bei der Edition der Arbeit hatte«, d.h., es bleibt auch unklar, ob wir es »überhaupt mit einem >authentischen<Seidel zu tun haben «(Voller 2017:84). Das Originalmanuskript gilt als verschollen; es ist demnach »unmöglich, nachzuvollziehen, wie tiefgreifend Prinzhorns Überarbeitung des Manuskripts ausgefallen ist « (ebd.: 70)..${ }^{22}$ Bereits der Sozialphilosoph Jürgen Frese, der Bewußtsein als Verhängnis 1975 für seine Antrittsvorlesung bearbeitet hat, ${ }^{23}$ spricht in Bezug auf Seidels

22 Prinzhorn schreibt im Vorwort, dass man sich »der Einsicht nicht verschließen [konnte], daß für eine Veröffentlichung die vorliegende Fassung keineswegs ohne weiteres verwendbar sei, wenn man dem Wertgehalt des Werkes zur Auswirkung verhelfen wollte. Von der urspünglichen Absicht, das Ganze oder Bruchstücke daraus mit allen Unvollkommenheiten einfach als >document humain $<$ zu veröffentlichen, mußte daher Abstand genommen werden « (Prinzhorn 1927: 8).

23 Frese ließ sein Manuskript später »trotz offenkundig erheblicher Mängel« drucken. Er betont in diesem Zusammenhang, dass weitere Publikationen zu Seidel und ein SeidelSammelband gescheitert seien (Frese 2001/o2: 61, Fn. 1). Wie Voller ausführt, fehlt Seidels 
Biografie von einer »dunklen Quellen-Sachlage« (Frese 2001/o2: 62, Fn. 5). Es sei »gut möglich, daß das im Text kondensierte Seidel-Bild hochgradig durchsetzt ist mit historisch fragwürdigen Konfabulationen Sohn-Rethels (mythisch verstärkt durch meine sicher selektiven Erinnerungen)« (ebd.). ${ }^{24}$ Ich werde Seidel im Folgenden trotz dieser Umstände selbst sprechen lassen. Da es mir analog zu den anderen Kapiteln dieser Arbeit - weder um die Intentionen des Autors noch um historische Vollständigkeit, sondern um die diskursiven Denkbewegungen jener Zeit geht, scheint mir dieses Vorgehen zulässig.

Seidels Text macht weniger den Eindruck, als ob darin Krisen diagnostiziert oder objektiviert, sondern als ob sie erfahren und durchlebt worden wären. Er legt am Anfang der Studie seine Skepsis offen, dass er möglicherweise keine letztgültigen >Lösungen< finden werde: Er gebe »keine fertigen Lösungen, sondern nur Lösungsversuche oder Andeutungen zu solchen, sowie neue Problemstellungen [...], soweit diese Arbeit überhaupt Wissenschaft sein will und nicht nur Anklage und Kritik« (Seidel 1927: 72). Ludwig Klages beginnt eine zeitgenössische Rezension von Bewußtsein als Verhängnis mit den Sätzen: »Bedeutung und Grenze des eigentümlichen Buches lässt sich mit dem einen Satze kennzeichnen: Es übt Kritik, scharfsinnig absprechende und oftmals treffende Kritik, ohne uns etwas Bejahenswürdiges nennen zu können« (Klages 1928: 629). Dass Seidels Buch kein Programm bereitstellt, wird auch von Voller betont: »Im Unterschied zu anderen Krisendiagnosen unterschiedlicher Couleur bietet Bewußtsein als Verhängnis kein Programm, dem es sich zu verschreiben lohnte, keine Rettung des im Untergang befindlichen Abendlandes, keine letzte Schlacht, die auf welchem Weg auch immer zu gewinnen sei, kein Versprechen einer kommenden Gemeinschaft« (Voller 2012: 323-324). Diese Alternativlosigkeit ist - laut Voller - einer der Gründe, weshalb Bewußtsein als Verhängnis Beachtung verdiene: Es reihe sich in die lange Reihe »alarmierender Krisendiagnosen « ein, ${ }^{25}$ um sich durch seinen

Name fast ausnahmslos »in den Biographien jener Denker, mit denen er in Kontakt stand, in den Kompendien, Überblickswerken und Einzelstudien zur (Heidelberger) Soziologie der Zwischenkriegszeit, zur frühen Kritischen Theorie und zur Geistes- und Intellektuellengeschichte der Weimarer Republik« (Voller 2012: 315). Einzige Ausnahmen sind Bernd Wirkus' Deutsche Sozialphilosophie in der ersten Hälfte des 20. Jahrhunderts (Wirkus 1996: 231-235) und Reinhard Laubes Karl Mannheim und die Krise des Historismus (Laube 2004: 418-422).

24 Gemäß Frese ist die einzige authentische Quelle von Seidels Biografie die Darstellung seines Lebenslaufes am Ende seiner Dissertation. Weiter gibt er als Quellen Prinzhorns Vorwort von Bewußtsein als Verhängnis und die mündlich überlieferten Erinnerungen Alfred Sohn-Rethels - eines Jugendfreundes Seidels - an (Frese 2001/o2: 62, Fn. 5).

25 Gemäß Christian Voller gehört Bewußtsein als Verhängnis in die lange Reihe »alarmierender Krisendiagnosen«, wie sie in den 1920er-Jahren erscheinen: Georg Lukács' 
»kompromisslosen Pessimismus « gleichzeitig von diesen zu unterscheiden: »Das Buch ist Ausdruck reiner Verzweiflung, der ohne Appell - und gewissermaßen folgerichtig in der lautlosen Selbststrangulation - verhallt; ein Aspekt, der vor allem ideen-, intellektuellen- und mentalitätsgeschichtliches Interesse verdiente« (ebd.: 323-324). Ein Text, der weder Alternativen noch »Identifikationsperspektiven « (Frese 2001/o2: 61) bereitstellt, sich aber dennoch intensiv mit den wissenschaftlichen Herausforderungen seiner Zeit auseinandersetzt, ist so selten wie aufschlussreich.

Ein weiterer Grund, weshalb Bewußtsein als Verhängnis gemäß Voller Beachtung verdient, liegt darin, dass Seidels Notizen »auf eigentümliche Weise den damals noch gültigen Zwischenstand einer geistesgeschichtlichen Entwicklung [konservieren], die von der gemeinsamen Erfahrung des Aufbruchs in der Jugendbewegung und einem geteilten Kanon literarischer und wissenschaftlicher Quellen zur Ausdifferenzierung und Aufspaltung in theoretisch wie politisch unversöhnliche Lager führte (Voller 2012: 324). Eine Gegenüberstellung von Seidels Text mit philosophiehistorisch epochemachenden Texten und Autoren erbringt den Befund, dass zeitbedingte Krisen und Herausforderungen für die Erneuerung der akademisch etablierten philosophischen Tradition einen zentralen Impetus darstellen. Ausgehend von einer Lektüre von Bewußtsein als Verhängnis lässt sich also in exemplarischer Weise aufzeigen, was in Texten, die sich primär mit Lösungen und Alternativen auseinandersetzen, meistens nur implizit vorhanden ist: die Problemkonstellationen, aus denen die Suche nach Alternativen erfolgt ist. Gleichzeitig kann deutlich gemacht werden, dass die von mir ausgewählten Philosophen ein bei Seidel auffindbares Dilemma (ich nenne es den Seidel'schen Circulus vitiosus) gar nicht erst aufkommen lassen: die Frage nach der Legitimität des eigenen Standpunkts. ${ }^{26}$

Im ersten Teil dieses Kapitels werden in knapper Form Einsichten in Seidels Schrift gewährt. Im zweiten Teil wird gezeigt, wie die zwar marginale, aber seit dem Erscheinen von Bewußtsein als Verhängnis sich kontinuierlich fortsetzende Rezeptionsgeschichte nahelegt, Seidels Text als ein extremes Beispiel für die gesellschaftliche und politische Krise der Weimarer Zeit zu verstehen. In einem dritten Teil schließlich versuche ich zu zeigen, dass Bewußtsein als Verhängnis als ein Beispiel für die Verschiebung philosophischer Themen in

Geschichte und Klassenbewußtsein (1923), Martin Heideggers Sein und Zeit (1927), Walter Benjamins Einbahnstraße (1928), Ludwig Klages' Der Geist als Widersacher der Seele (1929), Karl Jaspers Geistige Situation der Zeit (1931), Ernst Jüngers Arbeiter (1932) oder Max Horkheimers Wissenschaft und Krise (1932) (Voller 2012: 315).

26 Dieser Aspekt wird insbesondere im Kapitel zur Kritischen Theorie fokussiert. Im Fazit wird das Dilemma auf die Positionierung vorliegender Arbeit bezogen (siehe S. 198-206). 
andere Disziplinen angesehen werden kann: Der Text macht deutlich, dass sich die Philosophie am Anfang des 2o. Jahrhunderts einer Herausforderung ihrer Grundlagen gegenübersieht, indem er darlegt, dass die Inanspruchnahmen philosophischer Themen durch andere Disziplinen die Daseinsberechtigung der Philosophie im Allgemeinen infrage stellen.

\section{Unheilvolle Apologie auf die Wissenschaft: Alfred Seidels Circulus vitiosus}

»Wie kindlich optimistisch sind die Zeiten des Rationalismus in dem überschwenglichen Glauben an die Wirkungen des Erkennens; trotz aller Kritik ist noch heute das Pathos der Wissenschaft vielfach von diesem Glauben getragen « (Seidel 1927: 75). Seidel möchte in seinem Buch aufzeigen, inwiefern die zunehmende Bewusstwerdung wissenschaftlicher Zusammenhänge sowohl auf persönlich-individueller als auch auf gesellschaftlich-politischer Ebene zersetzend und zerstörend wirkt. »Wohlgemerkt greifen wir nicht den Wahrheitsgehalt der wissenschaftlichen Erkenntnisse an, sondern die Behauptung, dass sie ausschließlich die proklamierten Wirkungen auslösen « (ebd.). Angesichts der aus seiner Sicht problematischen Glorifizierung des wissenschaftlichen Fortschritts und des »Bewußtseins « stellt sich Seidel in seinem Buch wie bereits weiter oben erwähnt die Frage, »ob die Erkenntnis der sozialen und psychischen Vorgänge nicht gerade ihre Beeinflussung [der Erkenntnis, MD] unmöglich macht, zumindest hemmt - die Frage nach dem Bewußtsein als Verhängnis« (ebd.: 74).

Seidel zeigt in seinen Analysen, dass und inwiefern die zunehmende Bewusstwerdung naturwissenschaftlicher, psychologischer und soziologischer Zusammenhänge sowohl für das Individuum als auch für die Gesellschaft einen - mit Max Weber gesprochen - >entzaubernden< Effekt hat. Die »zunehmende Intellektualisierung und Rationalisierung «, so Seidel, steigere nicht die »echte allgemeine Erkenntnis der Lebensbedingungen, unter denen man steht « (ebd.: 8o). Sie bedeute im Gegenteil, »dass man alle Dinge - im Prinzip - durch Berechnen beherrschen könne« (ebd.). Unter dieser Voraussetzung untersucht Seidel die Bildung und Wirkung von Ideologien. Die Auswahl, die er diesbezüglich trifft, widerspiegelt zentrale Schlagworte der Zeit. Diskutiert werden der »Marxismus-Sozialismus«, die »Neuromantik« (unterteilt in: Gemeinschaft, Religion, das Kulturgewollte, die Natürlichkeit, das Erlebnis und die Frauenbewegung), der »Nationalismus«, der »Pazifismus« und schließlich auch die »Untergangsidee« im Sinne Oswald Spenglers (ebd. 166-202). Seidel könnte, so Jürgen Frese, problemlos als »Ahnherr« der Kritischen Theorie figurieren (Frese 2001/o2: 47). Voller nimmt diesen Aspekt 
auf und stellt Seidel explizit in den Kontext einer Vorgeschichte der Kritischen Theorie (Voller 2017). ${ }^{27}$

Dass Seidel jedoch auch die Wissenschaften, die Philosophie und vor allem seinen eigenen Standpunkt als Ideologien begreift, scheint ihn - im Unterschied zu Horkheimer und Adorno - in ein unerträgliches Dilemma zu treiben: Insbesondere im »Schluss « und im »Anhang « (»Bruchstücke aus anderen Schriften«) gleichen seine Gedankengänge einem Circulus vitiosus. »Da der Lebensfähige nur Illusionen als Weltbild haben kann, so ist dagegen der, der diese Illusionen zerstört, also der Wahrheitssadist, ein an sich lebensunfähiger, ein Selbstmördertypus, ein Instinktloser, ein Psychopath im üblichen Sinne« (ebd.: 220). Der Teufelskreis nimmt im Text immer dann seine Form an, wenn Seidel seine Analysen auf die Wissenschaft selbst bzw. auf seine eigene Stellung als Wissenschaftler rückbindet.

Seidels Dilemma korrespondiert mit dem Verlust einer >Außenperspektiveく. Denn da er sich selbst zum »Typus « des analytischen Wissenschaftlers, d.h. zu den »Wahrheitssadisten« zählt (ebd.: 31), arbeitet er ja auch selbst aktiv am individuellen und kulturellen Zerfallsprozess mit. Wie, so fragt sich Seidel, »kann man an dem eigenen Schopfe sich selbst aus dem Sumpfe ziehen" (ebd.: 212). ${ }^{28}$ Er sieht keine Möglichkeit, dem Dilemma zu entkommen: Es gebe »nichts Negativeres als das Gerede von der Positivität: der wahrhaft Positive redet nicht von ihr, braucht es auch nicht. Für den aber, der unter keinem festen Glauben steht, der also in diesem analytischen Geiste gefangen ist, was bleibt für ihn übrig, sofern er ein geistig aktiver Mensch ist? (ebd.: 203). Seidel demonstriert, dass ein solcher Mensch tatsächlich in die Bredouille gerät, denn: »Bewusster Nihilismus um des Nihilismus willen ist schon psychologisch nicht möglich, mit jedem Atemzuge verneint er diesen Nihilismus« (ebd.). Es bleibe also nur übrig, »den Nihilismus zu nihilisieren, all den Bewegungen, die unter einem Gerede von Posivität [sic!] de facto nur nihilistisch wirken, die Maske vom Gesicht zu reißen, sie zu entlarven « (ebd.). Man müsse »den Gegner in sich selbst auflösen, indem man seine eigene Theorie auf ihn anwendet« (ebd.). Seidel transponiert denselben Gedankengang auf den Bereich der Kultur. Es gälte, »den Kampf zu führen gegen die schamlose Selbstbejahung unserer Zeit um der Reinheit des Geistes und der wahren Kultur willen, sei sie auch in dieser Kultur nicht mehr möglich « (ebd.). Da für Seidel die »Reinheit des Geistes« in dieser Kultur nicht mehr zurückzuerlangen ist, besteht

$27 \quad$ Siehe Kapitel 5.1.

28 Diese Metapher wird im Fazit wieder aufgenommen. 
seine `Lösung« schließlich in einer »Nihilisierung des Nihilismus« (ebd.: 202). Man müsse »aus innerer Notwendigkeit negativ sein, aber gegen die Negativität, nihilistisch gegen den Nihilismus« (ebd.: 204). Der »Kampf« müsse somit »gegen den eigenen Typus « - gegen den »Wahrheitssadisten « - gerichtet sein, indem man »die Analyse gegen die Analyse treibt« (ebd.). Die »Sublimierung des Selbstmords«, schreibt Seidel, sei die »einzig mögliche psychische Grundlage richtiger Erkenntnisse« (ebd.: 214).

Seidel, so macht es den Anschein, reflektiert nicht nur andere wissenschaftliche Positionen, sondern versucht zugleich, seinen eigenen Standpunkt reflexiv zu erfassen. Er sucht nach Möglichkeiten, seine eigenen Gedanken und Überzeugungen zu legitimieren - und findet sie nicht. Seine Überlegungen fallen erkenntnistheoretisch gewissermaßen ins Bodenlose: Das Bewusstsein als eigenständige analytische Kategorie scheint aufgrund der Einsicht in andere Wissensformen immer wieder zu kollabieren. Mit anderen Worten: Seidels Text demonstriert die Unmöglichkeit, das Bewusstsein jenseits psychologischer und soziologischer Wirkungszusammenhänge zu denken oder zu behaupten. Folgendes Zitat verdeutlicht diesen Aspekt prägnant: »Die Entthronung der absoluten Ideen durch die Bejahung der Realitäten - heißen diese nun Leben, Macht, Sexualität einerseits oder Nation und Klasse andererseits - ist Zeichen der Auflösung der Kultur. Die Spannung zwischen Idee und Realität wird aufgehoben, die Realität selbst zur Idee erhoben [...] «(ebd.: 202). ${ }^{29}$ Das heißt, die Realitäten (im weitesten Sinne psychologische und soziologische Wirkungszusammenhänge) sind an die Stelle der absoluten Ideen (im weitesten Sinne des Bewusstseins bzw. der Philosophie) getreten. Realität (Psychologie/Soziologie) und Idee (Philosophie) fallen zusammen.

\section{Rezeption: Manifestation einer Krise}

Seidel, 1895 in Schlesien geboren, hat (nach einem Semester Medizin in Leipzig) in Freiburg, Berlin, Göttingen und Heidelberg Philosophie und Geschichte, später zusätzlich Nationalökonomie und Soziologie studiert. Vorlesungen besucht hat er u.a. bei Heinrich Rickert, Edmund Husserl, Ernst Troeltsch, Werner Sombart, Alfred und Max Weber, Karl Mannheim, Karl Jaspers und Ernst Bloch. Am Anfang der 1920er-Jahre lernte er Walter Benjamin, Siegfried Kracauer und Theodor W. Adorno kennen. ${ }^{30}$ In Freiburg war Seidel eine Zeit lang Mitglied einer Gruppe der freistudentischen Jugendbewegung. In Berlin schloss er sich dem Kreis rund um die als linksradikal,

29 Dieses Zitat wird zusätzlich auf S. 73 im Zusammenhang mit Husserls Selbstpositionierungen besprochen.

Zum Verhältnis zwischen Adorno und Seidel siehe S. 128-130. 
pazifistisch und antibürgerlich geltende Zeitschrift »Der Aufbruch $\aleph^{31}$ an, und in Heidelberg (1919-1922) bildete sich rund um den Ökonomen und Soziologen Emil Lederer im Anschluss an dessen Vorlesung über die Sozialtheorie des Marxismus eine zeitweise enge Arbeits- und Lebensgemeinschaft. In Heidelberg wurde Seidel in den Vorstand und zum Leiter der wissenschaftlichen Abteilung des Sozialistischen Studentenbundes gewählt. ${ }^{32}$ Seine von Alfred Weber betreute Dissertation Produktivkräfte und Klassenkampf ${ }^{33}$ kann laut Voller durchaus als eine Schrift begriffen werden, die mit Lukács' Geschichte und Klassenbewußtsein und Karl Korschs Marxismus und Philosophie die Entwicklung und Etablierung der Kritischen Theorie prägten (Voller 2017: 84).

Seidel bewegte sich in illustren intellektuellen Kreisen. Ohne jedoch ein autorisiertes Zeugnis seines Denkens hinterlassen zu haben (Voller 1912: 313), nimmt er sich 1924 das Leben. Wie erwähnt kommt der Psychiater und Kunsthistoriker Hans Prinzhorn Seidels Ersuchen, die Herausgabe seines Buches zu veranlassen, nach. In einer Mischung aus Paternalismus und Fürsorglichkeit zeichnet Prinzhorn das Bild eines Menschen, der in akademischer Hinsicht zwar scheitert, durch seine Redlichkeit aber »moralisch siegte« (Prinzhorn 1927: 20). Bezeichnend ist die Schilderung eines Vortragsabends im Kreise Karl Mannheims, der für Seidel »denkbar ungünstig« verlief; man habe eine »zerfahrene monologisierende Sprechweise« vernommen, »ein dürres Argumentieren « und ein »Zerreden von Tatsachen«, ohne »daß der einheitliche Sinn klar geworden wäre« (ebd.). »Immerhin haben einige Teilnehmer gespürt, daß sie hier einem ehrenvollen Unterliegen beiwohnten und daß ein Unzulänglicher, aber von einem reinen Müssen Getriebener moralisch siegte - beinahe noch wo er faktisch Unrecht hatte - nur dadurch, dass er ohne praktische Interessen seine Brust darbot und sein Wort aussprach" (ebd.). In Prinzhorns Vorwort wird Seidel als eine Person beschrieben, die sich mit absoluter Ehrlichkeit, ohne Berechnung und Kalkül einer vom Schicksal auferlegten Bestimmung hingibt und sich dem akademischen Kontext schutzlos

31 Nach vier Nummern wurde die Zeitschrift 1915 durch die Zensurbehörde verboten. »Anlass für das Verbot war ein Artikel [Ernst] Joëls >Von deutschen Hochschulen<, in welchem er den geist- und gehaltlosen Patriotismus der Studierenden und akademischen Soldaten anprangerte und sich für einen aristokratischen Bildungsbegriff Fichtes, Arndts und Kleists stark machte « (Bruns 2008: 217).

32 Dazu Seidel in einem Brief (1920): »Man presste mich als einziges Renommier-Goy in den Vorstand des sozialistischen Studentenbundes, und ich bin eingetreten, und zwar als formeller Leiter der wissenschaftlichen Abteilung, gerade wo meine Wissenschaft und mein Sozialismus, also besonders mein wissenschaftlicher Sozialismus zusammenbrechen« (Seidel 1927: 32).

33 Seidels Dissertation wurde erstmals 2008 unter dem veränderten Titel Produktivität und Klassenkampf gedruckt und mit einem Vorwort von Hugo Velardes versehen. 
ausliefert. So sei der Text Bewußtsein als Verhängnis »getragen von dem durchaus einheitlichen, aus gelebten Erfahrungen strömenden Geist des ehrlichen, tapferen Ringens um Sinn und um Form des kulturellen Lebens« (ebd.: 11). Der Grund, der es zur »Ehrenpflicht« mache, das Buch posthum herauszugeben, liegt für Prinzhorn in der »Persönlichkeit« Seidels. »Es sei nämlich Alfred Seidel ein ganz ungewöhnlich reiner und klar überschaubarer Repräsentant für eine Menschenart, die für das Gesicht dieser Zeit charakteristisch und von beträchtlicher struktureller Bedeutung ist « (ebd.: 3-4). Diese Bedeutung rühre nicht nur von Seidels »typische[r] Seins- und Denkart, sondern weil eine bestimmte Einstellung zu den allgemeinen Pähnomenen des Lebens, der Kultur, der Gesellschaft ihm konstitutionell vorgegeben war, in den Zeitströmungen sich schicksalsmässig vertiefte und mit der schon fast grauenhaften Konsequenz eines nihilistischen Amor fati durchgehalten wurde bis zum Untergang - ein Selbstopfer als Warnung an den Zeitgeist « (ebd.: 4).

Auch in den zeitgenössischen Buchbesprechungen wird der exemplarische Status von Seidel und seiner Schrift ausnahmslos hervorgehoben. In einer Rezension schreibt Kracauer Bewußtsein als Verhängnis den »Rang eines zeitgeschichtlichen Dokuments « zu, »denn die gegenwärtige Situation ist in ihm exemplarisch erfahren und entschieden verworfen worden. Verworfen von einem Denker, der sich den modernen Gedanken bis zur Selbstaufgabe ausgesetzt hat « (Kracauer 2011 [1927]: 521). Auch Margarete Susman betont, dass Seidel in seinem Buch »den Charakter gerade des Bewußtseins unserer Zeit« umreiße (Susman 1927: 338). Ernst Bloch schreibt einige Jahre später (1938) in einem literarisch motivierten Aufsatz, dass »der Student Alfred Seidel [...] nur scheinbar eine abseitige Type « sei. »Er drückt vielmehr aus, was manche damals gelitten haben« (Bloch 1965: 67). Sowohl Bewußtsein als Verhängnis als auch Seidels persönliches Schicksal werden in den zeitgenössischen Rezensionen als Manifestation einer unheilvollen Situation wahrgenommen: »Und so vollzog Seidel das Gericht über seine Zeit an sich selbst. Hier schweigt die Frage nach Wahrheit oder Irrtum « (Susman 1927: 341).

Die Begriffe Wahrheit und Wahrhaftigkeit kommen in den Besprechungen auffällig häufig vor. Von »redlichem Wahrheitswillen « und einer »nicht hoch genug zu schätzenden Ehrlichkeit« ist die Rede (Klages 1928: 629) sowie von einem »neuen Werther «, den ein »selber nicht Entzauberbares « zu Tode brachte (Bloch 1965: 71). Bloch schreibt weiter: »Der Freitod des Studenten Seidel aus Alt-Heidelberg zeigt [...] mit Zuspitzung, wie damals sehr vielen jungen Menschen der bürgerlichen Intelligenz das Licht entseelt, die Wahrheit zum Ekel geworden und gemacht worden war« (ebd.: 68). Im Zusammenhang mit seiner »Wahrhaftigkeit« verkörpere Seidel sogar die Möglichkeit zum Widerstand: Im Gegensatz zu »vielen Minderen seiner Art«, so Bloch, 
rettete er sich nicht aus dem »Nichts zu den Nazis«, um dort »gläubig und gefährlich« zu werden. Gleichzeitig sei er weder vom »Münchner Fasching« eines Klages noch vom »seherischen Georgekreis « verführt worden (ebd.: 69). Bei Bloch nimmt Seidel und insbesondere dessen Selbstmord zugleich die Wendung zum Hoffnungsträger: Im Vergleich mit Goethes Werther wird Seidel hier zu einem Nihilisten stilisiert, »der weiß, was das heißt « (ebd.). Gerade ein solch wirklicher Nihilismus könne möglicherweise »zur Arbeit am keimenden Leben« führen (ebd.: 71). Einzig Kracauer verurteilt Seidels Selbsttötung, indem er diesem »Schwäche« vorhält - eine Schwäche freilich, die »aus der Enttäuschung einer ganzen Generation « stamme. Die »Unzufriedenheit mit den herrschenden Wissenschaftsübungen« hätte Seidel nicht zur Absage an die »vernunftmäßige Bewußtheit« führen dürfen, sondern »zum Kampf für die Vernunft $\ll$. Seidel habe

sehr scharf gesehen, daß die »aufgeklärten« wissenschaftlichen Lehren unserer Zeit vielfach lediglich ein Deckmantel für die sich selbst setzenden Triebe sind [...]; aber er hat nicht gesehen, daß sie die Seele und das Leben darum allein zersetzen, weil sie sich gegen die Vernunft verstocken. Von einer Bewußtheit übermannt, die gar nicht die echte ist, hat der Zerquälte das Bewußtsein überhaupt als das Übel erachtet und vor ihm sich in die Nacht zurückgezogen. (Kracauer 2011 [1927]: 523-524)

Seidel wird also bereits von seinen Zeitgenossen als eine Art verkörperter Zeitgeist wahrgenommen. »Insgesamt wurde Seidels posthum erschienenes $>$ Hauptwerk also als eine eigentümliche Verknüpfung von Theorie, Lebensabschlussbericht und epochentypischer Verzweiflung gedeutet, der eine repräsentative Bedeutung zukomme« (Voller 2017: 71).

Seidels Rezeptionsgeschichte ist in spezifischer Weise mit einer Pathologisierung verknüpft, die Prinzhorn in seinem Vorwort etabliert, aber bereits in Seidels Analysen angelegt ist: »Wieweit ist die Geisteskrankheit Bedingung zu geistigem Schaffen und warum wird sie bei dem einen fruchtbar zum geistigen Schaffen verwandt und warum bei dem andern nur in körperlichen und seelischen Symptomen erschöpft? «(Seidel 1927: 213). Seidel nimmt die für das 19. und 20. Jahrhundert zentrale Problematisierung von >Genie und Wahnsinn in seine Gedankengänge auf. Unter der Überschrift Genie und Wahnsinn (zu Kurt Hildebrandt) schreibt er beispielsweise:

Wahnsinn oder psychopathische Erscheinungen seien mehr Folge als Ursache oder Bedingung. - Unsinn! Eine conditio sine qua non. Der psychopathische Zwang als direkter Antrieb zur Produktion. Überwindung der Unlust und Sinnlosigkeitsgefühle der psychopathischen Erscheinungen; das Werk als Äquivalent. Die Sublimierung des Selbstmordes; eine Tat, um vor dieser Flucht in 
den eigenen Tod (vor den Qualen der Psychopathie) sich zu retten, bestimmt oft auch inhaltlich die Probleme. (ebd.: 214) ${ }^{34}$

Rezeptionsgeschichtlich ist es bemerkenswert, dass in Bezug auf Seidel und seinen Text zwar eine pathologisierende Komponente mitgedacht, diese aber über das Individuum Seidel hinausgehend als gesellschaftliches Krisenphänomen rezipiert wird. »Die Objektivität einer kritischen Distanznahme sollte so gerade durch den radikalen, unbedingt negativen Subjektivismus des depressiven Außenseiters ermöglicht werden, der Seidel selbst war. Theorie und Praxis fallen damit in eins und speisen sich gleichermaßen aus Veranlagung und Lebensstil «35 (Voller 2017: 79).

Die Auslegung, Seidel als eine Art >verkörperten Zeitgeist $<$ zu betrachten die Seidel selbst mit der Feststellung, dass nicht er selbst, sondern der Weltgeist in ihm dachte (ebd.: 45), angelegt hat -, wird von Georges Siegmund in den 196oer-Jahren weitergetragen. Er führt Seidels »Lebensschicksal« im Rahmen seines Buches Sein oder Nichtsein. Die Frage des Selbstmordes als einen »Beleg« an, dass »der Nihilismus in letzter Konsequenz bei der Selbstzerstörung endet [...]« (Siegmund 1970: 358). Seidel wird hier letztlich als ein typischer Fall für das »Grundleiden unserer Zeit, den Nihilismus« dargestellt (ebd.: 378). »Seidel gerät in den Sog einer Geistesströmung, welche in mächtiger Welle die nachkantische Philosophie und Weltanschauung beherrscht, wonach die Wahrheitsfrage der Religion endgültig verrammelt und nur noch eine entlarvende Psychologie möglich erscheint $[. ..] \ll($ ebd.: 367).

Eine politische Funktion nimmt Bewußtsein als Verhängnis 1979 in einer von Alfred Sohn-Rethel angeregten Neuauflage ein. ${ }^{36}$ Im Vorwort werden Seidel und sein Buch mit der linken politischen Bewegung der 1970er- bzw. 1980er-Jahre in einen Zusammenhang gebracht. ${ }^{37}$ W. Meier - der Verfasser des

34 Dem »Genie«, sagt Seidel in diesem Zusammenhang, sei es gelungen, »seine Psychopathien in seinen Lebensplan einzustellen und nicht den verflachenden Gesundmachern anheimzufallen [...]« (Seidel 1927: 214). Die gegenwärtige »Ideologie der Gesundheit und Natürlichkeit und der bewusste Kampf gegen die Psychopathien« sei einer der Gründe für die Seltenheit von »Genies in unserer Zeit« (ebd.).

35 Seidel berichtet über seine eigenen Depressionen in seinen Briefen. So etwa 1923: »Das Schrecklichste sind die durch keine äußeren Ursachen veranlassten endogenen Depressions- und Apathie-Zustände; im allgemeinen aber geht es mir psychisch bedeutend besser. Ich lasse mich nicht unterkriegen; auch kann ich sehr zufrieden sein, daß ich Menschen habe, die sich rührend um mich kümmern[,] und ich keineswegs mich als verlassen betrachten muß (Prinzhorn 1927: 33).

36 Seidel, Alfred (1927 [1979]: Bewußtsein als Verhängnis, herausgegeben und mit einem Vorwort versehen von W. Meier (d.i. Helmut Höge), Bremen: Impuls Verlag.

37 Durch die zunehmenden intellektuellen und künstlerischen Produktionen und Rezeptionen habe in der linken Bewegung eine Entwicklung eingesetzt, die soziale 
Vorwortes - beschreibt Seidel zwar als einen "politischen Seismographen«, der bereits Mitte der 1920er-Jahre »eine politische und soziale Entwicklung in Deutschland heraufziehen [sah], die Jahre später alle revolutionären Anstrengungen zur Sinnlosigkeit verurteilte«, strebt aber in keiner Weise an, aus Seidels Ausführungen theoretische Schlüsse irgendeiner Form zu präsentieren. Vielmehr wird hier der Seidel'sche Circulus vitiosus absichtlich vorangetrieben: Bewußtsein als Verhängnis werde »keine wesentlich neuen Erkenntnisse vermitteln (darin liegt ja gerade die Aktualität dieses Buches begründet), allerhöchstens, dass ihnen [den Gruppen und Zirkeln, MD] die Lektüre eine gewisse Genugtuung bereiten wird « (Meier 1979: o.S.). Seidels Projekt - das Bewusstsein mit dem Instrumentarium des Bewusstseins anzugehen - sei »weder unsinnig noch besonders neu: nicht >wer spricht?<, sondern >von wo aus« wird gesprochen? « (ebd.). Im Versuch, diese Frage zu beantworten, »könne natürlich das Problem auf den so Fragenden zurückschlagen« (ebd.). Seidel beantworte »die Frage damit, dass er Selbstmord begeht« (ebd.). Der Unsinn und die im Grunde pervertierte Sinnlosigkeit, ein Buch mit dem Titel Bewußtsein als Verhängnis überhaupt zu vertreiben bzw. zu lesen, wird zum Mittel für politischen Aktivismus. Seidel nimmt hier, wenn auch in anderer Form als in den bisherigen Rezeptionen, eine exemplarische Rolle ein: Er wird zum intellektuellen Vorzeige-Anarchisten für die Diskussionen der 198oer-Jahre stilisiert. ${ }^{38}$

Eine Besonderheit von Seidels Wirkungsgeschichte ist, dass sein Denken nicht von seinem Leben und Sterben loszulösen ist. Sein Denken zeichnet sich »auf eigentümliche Weise dadurch aus, die eigene Erfahrung und Stellung

Auseinandersetzungen immer mehr ausklammere. »Diese Entwicklung steht natürlich in dem engsten Zusammenhang mit der Profilierung eines Teils der ehemals aktiven Linken als Intellektuelle oder Künstler-Persönlichkeiten, die biographisch genau das zum Programm erheben, was sich der Leninist noch nicht einzugestehen wagte: die individuelle Karriere« (Meier 1979: Vorwort, o.S.). Diese »Hinwendung der Linken zur schönen Literatur« werde beispielsweise im Prinzip Hoffnung - »diesem scheißpathetischen Lyrismus « - manifestiert. In Bewußtsein als Verhängnis hingegen gehe es »um eine grundsätzliche Auseinandersetzung mit der Bedeutung [...] des `logos« (ebd.).

38 Ein weiterer Bericht zu Seidel aus dieser Zeit stammt von Bettina Wassmann, Bremer Buchhändlerin: In Bezug auf die von ihr initiierte Festschrift L'invitation au voyage von 1979 für Alfred Sohn-Rethel erzählt sie: »Da ist ein Text dabei über Alfred Seidel, das war ein alter Freund von Alfred aus der Heidelberger Studienzeit, also aus den 2oer-Jahren, dieser Alfred gehörte damals schon in die Prinzhorn-Therapie, weil er unter schweren Depressionsattacken litt. Sohn-Rethel sagte immer, er habe nie jemand Schlaueren kennen gelernt, und das ohne jede Sinnlichkeit. Und der schrieb mit 23 Jahren ein Werk, das hieß >Bewusstsein als Verhängnis`« (Goettle 2005: 15-16). Darauf hätten die Gäste schallend gelacht, und Wassmann fuhr fort: »Alfred liebte ihn sehr. Eines Tages hat sich Alfred Seidel das Leben genommen. Und wisst ihr, wo? Auf dem Bahnhofsklo!« (ebd.). 
innerhalb der Gesellschaft zum Ausgangspunkt umfassender Zeitdiagnostik zu machen « (Voller 2017: 63). Insbesondere seine Selbsttötung unterstützt, dass Bewußtsein als Verhängnis als exemplarisch für die politische und gesellschaftliche Krise der Weimarer Zeit wahrgenommen wird. »Seidels Selbsttötung [...] trug Züge eines Fanals insofern, als sich objektive Krisentendenzen und individuelles Triebschicksal in ihr wechselseitig durchwirkten « (Voller 2012: 322-323). In diesem Zusammenhang steht auch die Feststellung, dass die Ahnung von Seidels Bedeutung »arkan« vermittelt werde (Frese 2001/o2: 47). ${ }^{39}$ »Arkan, das bedeutet, nicht nur abseitig und verborgen, sondern auch heimlichtuerisch und kultisch. Es schwingt da ein Ton mit, der sich dem Andenken allzu jung, zumal durch eigene Hand, Verstorbener leicht beimischt, und der stets etwas von Heiligenverehrung an sich hat« (Voller 2012: 316). Vollers Anliegen, diese Wirkungsgeschichte nicht blindlings fortzuschreiben, sondern sie selbst thematisch zu machen, wird hier geteilt: Seidel wird nicht als Prophet oder Philosoph verstanden, der die >Wahrheit< jener Zeit in tragischer Weise erfasst hat, sondern er wird als ein Autor gelesen, der zu verstehen hilft, mit welchen Herausforderungen sich Wissenschaftler und Wissenschaftlerinnen und insbesondere Philosophen und Philosophinnen in dieser Zeit konfrontiert sahen.

Gleichwohl fungiert die Figur Seidel im vorliegenden Buch nicht quasi als Antithese zu den Philosophen oder als Anti-Philosoph: An Seidel und seinem Text und an der Art und Weise, wie Biografie und Schrift kolportiert wurden, kann zugleich eine Art philosophische Wahrheitsevokation beobachtet werden: Gerade aufgrund seines >Scheiterns - exemplifiziert durch seine Biografie - erzeugt er Bedeutung und Sinn. ${ }^{40}$ Was Voller diesbezüglich als »Heiligenverehrung« bezeichnet, korreliert in der hier eingenommenen Perspektive mit der Suggestion, dass Seidel explizit für die Wahrheit gelebt hat und gestorben ist. Dies wird trotz oder wegen, jedenfalls in Kombination mit Tragik positiv kolportiert. Am deutlichsten drückt dies die Journalistin Susman aus: »Ganz bewusst wollte er [Seidel] durch diese Tat Sinn schaffen, den Sinn seines Lebens erhärten: die Analyse nicht nur für eine Person, sondern auch für seine Generation überwinden, indem er ihre zerstörende Wirkung an seiner eigenen Person aufwies« (Susman 1927: 341). Kurz: Seidels Biografie befördert die Auffassung oder Fiktion, dass er im Auftrag der Wahrheit geschrieben habe und gestorben sei: Er hatte, so wird suggeriert, keine andere Wahl. Dies

39 Bewußtsein als Verhängnis sei »ein geschmäcklerisch so genannter 〉Geheimtip« (Frese 2001/02: 47).

40 Der zentrale Aspekt, dass Seidel und sein Text eine spezifische Form philosophischer Wahrheit erzeugen, wird im Kapitel 5.4 wieder aufgegriffen (S. 180-188). 
wiederum verstärkt den Eindruck, dass das Bewusstsein (und in der hier vorgenommenen Übertragung: die Philosophie), wie es Seidel versteht, tatsächlich an einem Endpunkt angelangt ist. Seidels Rezeptionsgeschichte macht deutlich, dass philosophische >Wahrheiten innerhalb des philosophischen Diskurses oft personifiziert in Erscheinung treten. Wie erwähnt ist in keiner anderen wissenschaftlichen Disziplin die personenzentrierte Deutungsmacht derart ausgeprägt wie in der Philosophie (Schnädelbach 1983: 120). Dies verweist darauf, dass die Rückbindung der Philosophie an konkrete Personen für philosophische Erneuerungsprozesse ein notwendiges Kriterium zu sein scheint.

Sowohl die Person Seidel als auch sein Buch Bewußtsein als Verhängnis werden in der Literatur also als ein Beispiel für die kulturelle und politische Krise der Weimarer Zeit rezipiert. Diese Rezeption kann um einen weiteren Aspekt ergänzt werden: Seidels Schrift lässt sich zusätzlich als ein Beispiel für die Schwerpunktverlagerung philosophischer Themengebiete in andere Disziplinen verstehen. Erstens wird in Bewußtsein als Verhängnis greifbar, dass am Anfang des 2o. Jahrhunderts das Thema des Bewusstseins für die Naturwissenschaften, die Psychologie und die Soziologie an Bedeutung gewinnt. Und zweitens wird in diesem Text deutlich, dass die Verschiebung philosophischer Fragestellungen in andere disziplinäre Felder für die Philosophie problematisch und krisenbehaftet war.

\section{Verlust disziplinärer Standpunkte}

Seidels Schrift, so betont Frese, besitze eine "philosophiehistorisch bemerkenswerte Bedeutung « (Frese 2001/o2: 47). »Für die zukünftige Philosophiehistorie des 2o. Jahrhunderts (und für eine ganz unengagierte Ideologiekritik) bildet Seidel ein aufschließendes extremes Phänomen [...] « (ebd.: 61). Hier führe »ein Autor mit sich selbst ein Experiment durch [...], das ein Bündel von zusammengehörigen Theoremen so von den Bedingungen seiner ideologischen Rezipierbarkeit isolierte, dass eine nahezu ideologiefreie, aber zugleich unvollziehbare Synthese entstand [...]« (ebd.). Frese begreift Seidel und seinen Text folglich als eine Folie zur Untersuchung zeitgenössischer Theoriebildungen: An den jeweiligen Abweichungen vom Seidel'schen Idealtypus ließen sich Stellen identifizieren, wo diese Theorieströmungen »wesentlich Ideologien sind [...] « (ebd.). In der hier eingenommenen Perspektive ist nicht Freses Trennlinie zwischen Ideologien und Nichtideologien relevant, sondern seine Feststellung, dass Seidel für die Philosophiegeschichte des 20. Jahrhunderts eine Bedeutung besitze. Diese Bedeutung wird auch von Voller betont: »Bewußtsein als Verhängnis bietet nicht nur die Möglichkeit, das Zerbrechen und Auseinanderdriften der Kultur- und Sozialphilosophie seit Mitte 
der 1920er Jahre nachzuvollziehen, sondern könnte auch zum Ansatzpunkt für eine synthetisch orientierte Aufarbeitung dieser Geschichte genommen werden « (Voller 2012: 325). Diese Aufarbeitung wird hier zwar nicht, oder nur sehr punktuell, geleistet, die Beobachtungen von Frese und Voller stützen aber das Vorgehen, in Bewußtsein als Verhängnis philosophieinterne Krisen auszumachen, die für philosophische Erneuerungsansprüche in den 1920er-Jahren konstitutiv sind.

Seidel bezeichnet sich selbst, wie erwähnt, nicht als Philosoph, noch spricht er explizit von der Philosophie. Mit seinem Thema aber - mit der Frage nach dem Bewusstsein - begibt er sich in das philosophische Kernthema per se. Gerade weil er sein Themengebiet nicht vorgängig disziplinär eingrenzt, ist sein »philosophisches Durcharbeiten « (Voller 2012: 324) so aufschlussreich. Es offenbart dadurch nicht nur den »Zwischenstand einer geistesgeschichtlichen Entwicklung « (ebd.), sondern den einer philosophieinternen: Insbesondere die bereits zitierten Schlusspassagen aus Bewußtsein als Verhängnis verdeutlichen, wie die Erkenntnisse aus neu entstehenden Disziplinen traditionell philosophische Topoi infrage stellen: etwa die Identifikation von Wahrheit mit dem >reinen< Geist (darauf reagiert vor allem Husserl), die Infragestellung der Metaphysik als einer Wissenschaft (darauf reagiert vor allem der Wiener Kreis) oder die positive Wertung einer fortschrittlichen Vernunft (darauf reagiert vor allem die Kritische Theorie).

Ein genauer Blick auf Seidels Fragestellung hilft zu verstehen, vor welchen Herausforderungen Philosophen und Philosophinnen zu Beginn der 1920erJahren stehen, wenn sie den Wahrheitsanspruch ihrer eigenen Disziplin neu zu begründen und zu legitimieren versuchen: Auf der einen Seite stellt Seidel die Frage nach dem Bewusstsein ausgehend von den Erkenntnissen der neu entstehenden Wissenschaften: »Wir stehen also mit den Verteidigern der Wissenschaft auf dem Standpunkt, dass die Welt des Sozialen und Psychischen - innerhalb gewisser Grenzen - erkennbar sei, und dass schon vieles erkannt wurde « (Seidel 1927: 74). Auf der anderen Seite wiederum stellt er die Frage nach der Reflexion auf diese Wissenschaften: $»$ Wir stellen demgegenüber die Frage, ob die Erkenntnis der sozialen und psychischen Vorgänge nicht gerade ihre Beeinflussung unmöglich macht, zumindest hemmt - die Frage nach dem Bewußtsein als Verhängnis« (ebd.). In der hier angestrebten Lektüre können in Seidels Text zwei Ebenen der Analyse unterschieden werden: Einerseits stellt er seine Frage nach dem Bewusstsein ausgehend von den Erkenntnissen aus anderen Wissenschaften, andererseits aber hat er den Anspruch, diese Erkenntnisse zu reflektieren bzw. zu kritisieren. Er nimmt, mit anderen Worten, (neue) Erkenntnisse aus anderen Wissenschaften in seine Reflexionen über diese Wissenschaften auf. Aber er >scheitert< an diesem Anspruch. Denn 
von welcher Warte aus - so das Dilemma - können wissenschaftliche Erkenntnisse kritisch reflektiert werden, wenn sie zugleich umstandslos als gültig anerkannt werden? ${ }^{41}$ Seidels Circulus vitiosus kann folglich dahingehend interpretiert werden, dass er keine philosophische Position einnimmt, von der aus er eine metawissenschaftliche Reflexion vollziehen könnte. Seine Analysen verfangen sich beständig in naturwissenschaftlichen, psychologischen und soziologischen Begründungsversuchen, ohne dass diese stringent durchgeführt werden könnten (Wirkus 1996: 235). Die Krisen, die in Bewußtsein als Verhängnis manifest werden, sind die Verluste genuin philosophischer Standpunkte infolge der Erkenntnisse aus anderen Wissenschaften. Seidels Schrift verdeutlicht in diesem Zusammenhang, dass philosophisches Denken prekär sowie unsicher ist und sich innerhalb des Systems der Gesamtwissenschaften immer wieder neu zu behaupten hat.

Seidel, dies sei erneut betont, beschreibt diese Krisen nicht in systematischer oder objektivierter Weise. Die Krisen äußern sich anhand seiner Thematik, seines Schreibstils und seiner Gedankengänge, in denen er die Reflexion auf sein eigenes Denken nicht außen vor lässt. Der Text ist einerseits an den akademischen Gepflogenheiten seiner Zeit orientiert, bricht diese andererseits aber an genau jenen Stellen, wo wissenschaftliche Wahrheitsansprüche hinterfragt werden. Diese Eigenheit auf der textuellen Ebene vermag es, genau jene Unsicherheiten, Brüche und Widersprüche sichtbar zu machen, von denen in der Philosophie häufig nur als Gegenstand die Rede ist. Indem der Text immer wieder an seinen eigenen Ansprüchen scheitert, stellt er aus, dass philosophisches Denken fragil und gefährdet sein kann.

In den nachfolgenden Kapiteln wird an unterschiedlichen Beispielen dargelegt, wie Philosophen diese Prekarität in Anspruch nehmen, um philosophische Standpunkte argumentativ zu behaupten bzw. neu zu etablieren. Wie deutlich geworden ist, sind Philosophen und Philosophinnen am Anfang des 2o. Jahrhunderts mit der Aufgabe konfrontiert, jene Erkenntnisse aus anderen Wissenschaften aufzunehmen und zu reflektieren, die die Existenzberechtigung der Philosophie infrage stellen, und sie sind gleichzeitig gefordert, die Existenzberechtigung der Philosophie als eigenständige Disziplin neu zu begründen. Indem sie ihre eigene Position mit den neuen Herausforderungen aus anderen Wissenschaften anreichern, delegitimieren sie die Infragestellung

41 Voller schreibt, dass sich Seidel, wie die Autoren der Dialektik der Aufklärung, in eine argumentative Bredouille manövriere: »Von welcher Warte aus kann derjenige Kritik am wissenschaftlichen Fortschritt üben, der weder Feind des Fortschritts ist, noch grundsätzlich leugnet, dass der wissenschaftliche Fortschritt gültige Erkenntnisse liefert, also tatsächlich Fortschritt ist?« (Voller 2012: 318). 
ihrer eigenen Disziplin und evozieren dadurch neue, genuin philosophische Standpunkte. Das heißt, es wird sich zeigen, dass die untersuchten Philosophen sich gezielt mit den Angriffen aus anderen Wissenschaften auseinandersetzen und diese Auseinandersetzungen dafür nutzen, historisch gewachsene Selbstverständnisse der traditionellen Philosophie zu reaktivieren. Es wird sich auch zeigen, dass die daraus enstehenden Neupositionierungen untrennbar mit einem Anspruch auf Allgemeingültigkeit und Objektivität einhergehen. Besonders kompakt werden diese Zusammenhänge im disziplinären Selbstverständnis der Philosophie an einer Textstelle des Neukantianers Heinrich Rickert ersichtlich. Bevor im nächsten Kapitel als erstes Beispiel Husserls Selbstpositionierungsstrategien näher umrissen werden, lohnt ein kurzer Exkurs zu dieser Textstelle - erwähnt sei auch, dass Seidel bei Rickert Vorlesungen besucht hat und diesen als "raffinierten Impressionisten « (Prinzhorn 1927: 54) bezeichnet haben soll, aber Distanz wahrte: »Von Rickerts schön gefügtem System und seiner bestechenden Dozierweise ließ er sich keineswegs fangen $\ll($ ebd. $) .42$

\section{Das Weltganze zum Gegenstand machen}

In seinem Aufsatz »Vom Begriff der Philosophie« fragt Rickert: »Weshalb reden die Philosophen so viel von dem Begriff ihrer Wissenschaft, statt wie andere Forscher an die Bearbeitung ihrer Probleme zu gehen?« (Rickert 1910/11: 1). Rickert gibt folgende Antwort: Weil die Philosophie »das Ganze zu ihrem Gegenstande zu machen hat«, sich dieses »Ganze« aber aufgrund der ständigen Entwicklung der Einzelwissenschaften immer wieder verändere, müsse die Philosophie auch laufend sich selbst neu bestimmen und definieren. »Da die Spezialforschung Teile desselben Ganzen behandelt, muss mit ihrer Entwicklung und Ausbreitung der Begriff der Philosophie, die ursprünglich alle Wissenschaften einschloss, wechseln, und außerdem lassen sich nur von Teilen der Welt vor ihrer eingehenden Untersuchung Begriffe bilden, die bestimmt genug sind, um das Gebiet einer Wissenschaft eindeutig abzugrenzen« (ebd.). Die Einzelwissenschaften behandeln gemäß Rickert also jeweils einen Teil der Welt, die Philosophie jedoch die Welt in ihrer Totalität: »Was das Weltganze ist, gehört zu den Fragen, die erst von der Philosophie selbst beantwortet werden können « (ebd.). Dieser Gedankengang Rickerts zeigt beispielhaft, dass erst durch die Verhältnisbeschreibung

42 »Er [Seidel, MD] fühlte deutlich die journalistisch-popularphilosophische Routine, mit der R. [Heinrich Rickert, MD] die philosophiegeschichtliche Leistung seines Lehrers Windelband zu Kollegsensationen aufzog. Er spürte das verhängnisvolle Vorbeigehen an der Problematik« (Prinzhorn 1927: 54). 
zu den Einzelwissenschaften ein bestimmtes philosophisches Selbstverständnis erzeugt wird: Die Philosophie ist jene Wissenschaft, deren Gegenstand das »Weltganze« ist. Rickerts Frage deckt sich mit einem Interesse der nachfolgenden Kapitel: Warum reden Philosophen so viel von ihrer eigenen Disziplin? Welche Funktion hat diese Rede ${ }^{43}$ Rickerts Antwort jedoch weicht in drastischer Weise von der hier eingenommenen Perspektive ab: Wo er aus der Frage eine Bestimmung für die Philosophie ableitet, nämlich die Welt in ihrer Ganzheit zu erfassen, soll es hier um die Funktion solcher Bestimmungen für die Selbstdefinition der Philosophie gehen. Rickerts Gedankengang wird hier also gewissermaßen umgedreht: Durch ihre gezielte Auseinandersetzung mit anderen Wissenschaften, so die argumentative Stoßrichtung, aktualisieren Husserl, die Vertreter des Wiener Kreises sowie Horkheimer und Adorno je Positionen, die vorgeben, das »Weltganze« erfassen zu können.

43 Dass Philosophen das Selbstverständnis ihrer eigenen Disziplin zum Gegenstand von philosophischen Diskussionen machen, gehört selbst wiederum zum Selbstverständnis der Philosophie. »Manchmal scheint deren Arbeit [der Fachleute, MD] sich entsprechend im Streit um das Selbstverständnis der eigenen Wissenschaft zu erschöpfen« (Lembeck 2010: 13). 